\title{
Research on Data Generation Method for Train Control System
}

\author{
Yu Tian ${ }^{1, a}$, Hai-feng Wang ${ }^{2, b}$ and Quan-xin Sun ${ }^{1, c}$ \\ ${ }^{1}$ School of Traffic and Transportation, Beijing Jiaotong University, Beijing 100044, China; \\ ${ }^{2}$ School of Electronic and Information Engineering, Beijing Jiaotong University, Beijing 100044, \\ China. \\ a14120886@bjtu.edu.cn, bhfwang@bjtu.edu.cn, 'cqxsun@bjtu.edu.cn
}

Keywords: Train Control System, Data, Method.

\begin{abstract}
Train control system can achieve automatic control in the whole process or the part of process. The data of train control system in role is irreplaceable. Keeping train control system's data transmission and exchange real-time and accurate, can make train safe. Completing the automatic generation of train control system's data, can supply the train control system with safe and reliable sources of data. With the help of the train control system, the train will be more safe and stable. This is also the meaning of this research.
\end{abstract}

\section{Introduction}

In order to establish a train operation control system which can completely balance both normal-speed railway and the high-speed railway. Chinese railway ministry thought about the existing domestic technology level, referred to the foreign train control system specification and experience, combined with the characteristics of China's railway transportation and followed the principle of unified planning of railway. Then they set up Chinese train control system in 2002. CTCS is short for Chinese train control system. The main function of CTCS is to ensure the driving safety and not disturb the normal driving of the locomotive crew. The goal of CTCS is to further improve the safety of train operation, further strengthen the system design, match the international standards and meet the needs of transportation development. Combining the situation of our country, starting from the actual demand, and according to the principle of unified design of on-board and ground equipment, CTCS is divided into CTCS-0, CTCS-1, CTCS-2, CTCS-3 and CTCS-4 according to the structure and function of the system[1]. This research mainly talks about CTCS-3 train control system.

\section{CTCS-3 Train Control System}

Introduction of CTCS-3 Train Control System. CTCS-3 train control system has been adopted as the unified technology platform for $350 \mathrm{~km} / \mathrm{h}$ high speed railway in China. It is an important part of Chinese railway equipment and technology system. It is also the core technology which can make high-speed train reliable, efficient and safe. CTCS-3 train control system is based on global system for mobile communication for railway (GSM-R) technology which can achieve the exchange of information between on-board and ground equipment, and let radio block center (RBC) generate moving permit (MA) and sent it to train control equipment of train control system. It uses a lot of advanced technology to protect high-speed train, including the interval of train and the speed of train. Real-time monitoring and overspeed protection are core technology. CTCS-3 train control system uses one-time braking mode which is under target-distance mode. The braking equipment is prior to human control. Because of the compatibility of the system, CTCS-3 train control system allow train to go across different lines which are different speed level.

Component of CTCS-3 Train Control System. CTCS-3 train control system includes on-board equipment and ground equipment. On-board equipment includes GSM-R remote terminal unit (RTU), vehicle safety computer (VC), transponder information transmission module (BTM series), track circuit information receiving unit (TCR), human machine interface (DMI), train interface unit (TIU) 
and recording unit (JRU/DRU) etc. Ground equipment includes station train control center (TCC), radio block center (RBC), responder (including Lineside Electronic Unit), GSM-R communication interface equipment and ZPW-2000 series of (UM series) track circuit, etc. The structure of CTCS-3 train control system is shown in Figure 1.

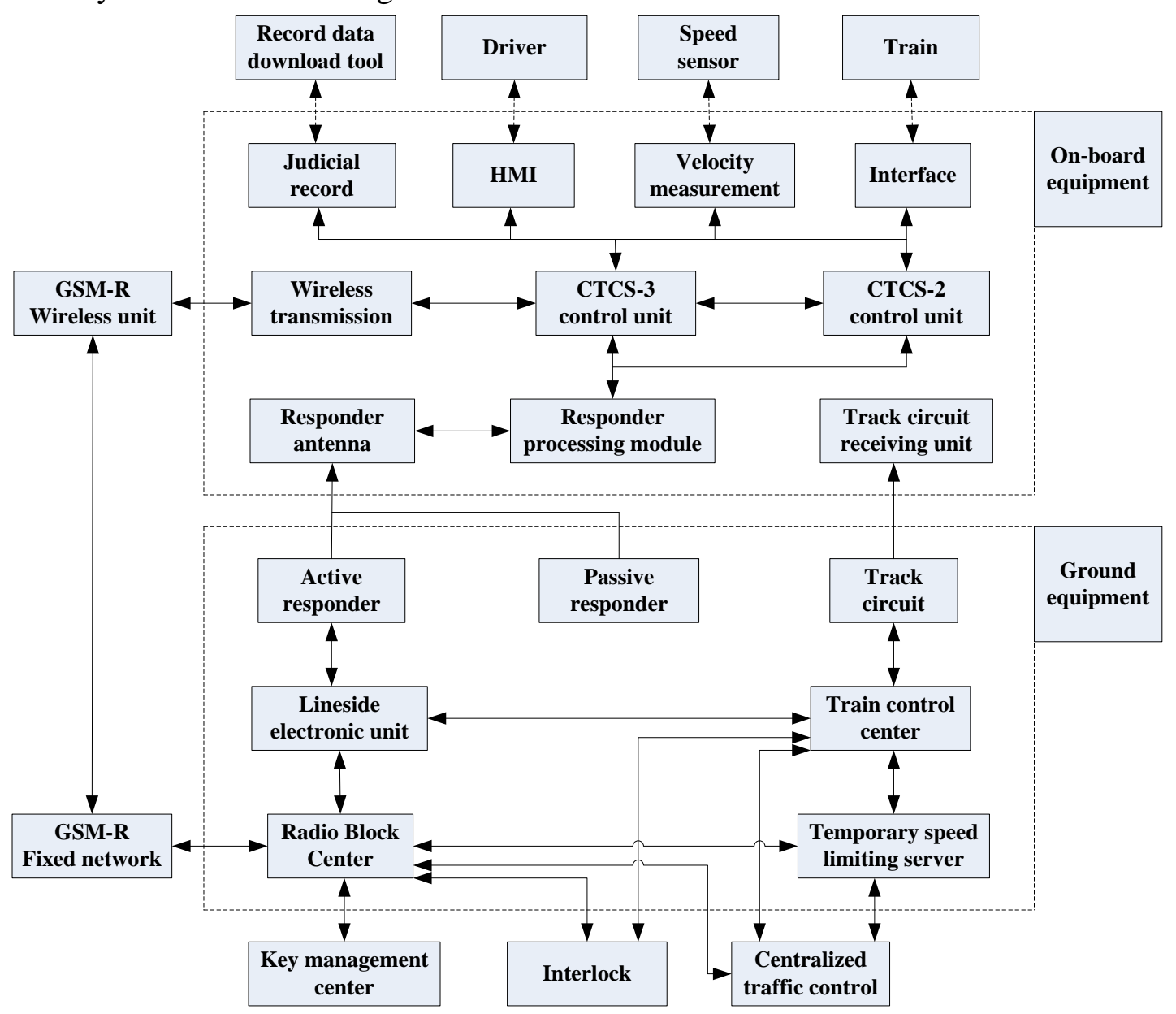

Fig. 1 The structure of CTCS-3 train control system

\section{Demand Analysis of Train Control System's Data}

Overview of Train Control System's Data. Train control system data is provided by the railway administration and research institute which designs railway signal system[2]. They put these data into the train control system database and regard it as the main basis of train control system data. The train control system data covers three aspects: the station engineering manual, the ground equipment information table and the train control engineering data table.

Requirements for Train Control System's Data. What makes train control system controlling the train safely is that the basic data from the train control system database. The requirements of the train control system's data have the following three points: accuracy, completeness and standardization.

Accuracy means: In the CTCS-3 train control system, system uses one-time braking mode which is under target-distance mode. And the length of the front section is one of the most important information of train control system's data. It can make the system controlling the train more safely. If the information of length of the front section have big errors with the actual length of section, it will directly endanger the running safety of train.

Completeness means: The data of train control system mainly include the line information of section which is controlled by the CTCS-3 train control system. When the CTCS-3 train control system is abnormal or the train arrives at the degraded section, the system should switch to the lower level of train control system. After the system switched the system level, The system should ensure 
the safety of the running train. So except the switching point of train control system and the lower level of train control system, the train should be controlled by the CTCS-3 train control system in the rest sections. So the line information including speed, slope, line sections and a series of information must be generated and putted into train control system database.

Standardization means: Train control system's data is regarded as the input data of transponder or no source transponder, station of train control center and a series of ground equipment. In the process of preparing the train control system's data, we must pay attention to the data standards and standardization of data. Such as the signal machine's name, the unit of line's length and the kilometers of road marking. They all should be in one standard.

Component of Train Control System's Data. The data of train control system covers three major categories: the station engineering manual, the train control engineering data tables and other data tables. The station engineering manual and the train control engineering data tables which are provided by the railway signal department and research institute are the most important components of the train control system's data. The other data table is compiled by the manufacturer of the train control system. The classification and main structure of train control system's data are shown in Figure 2.

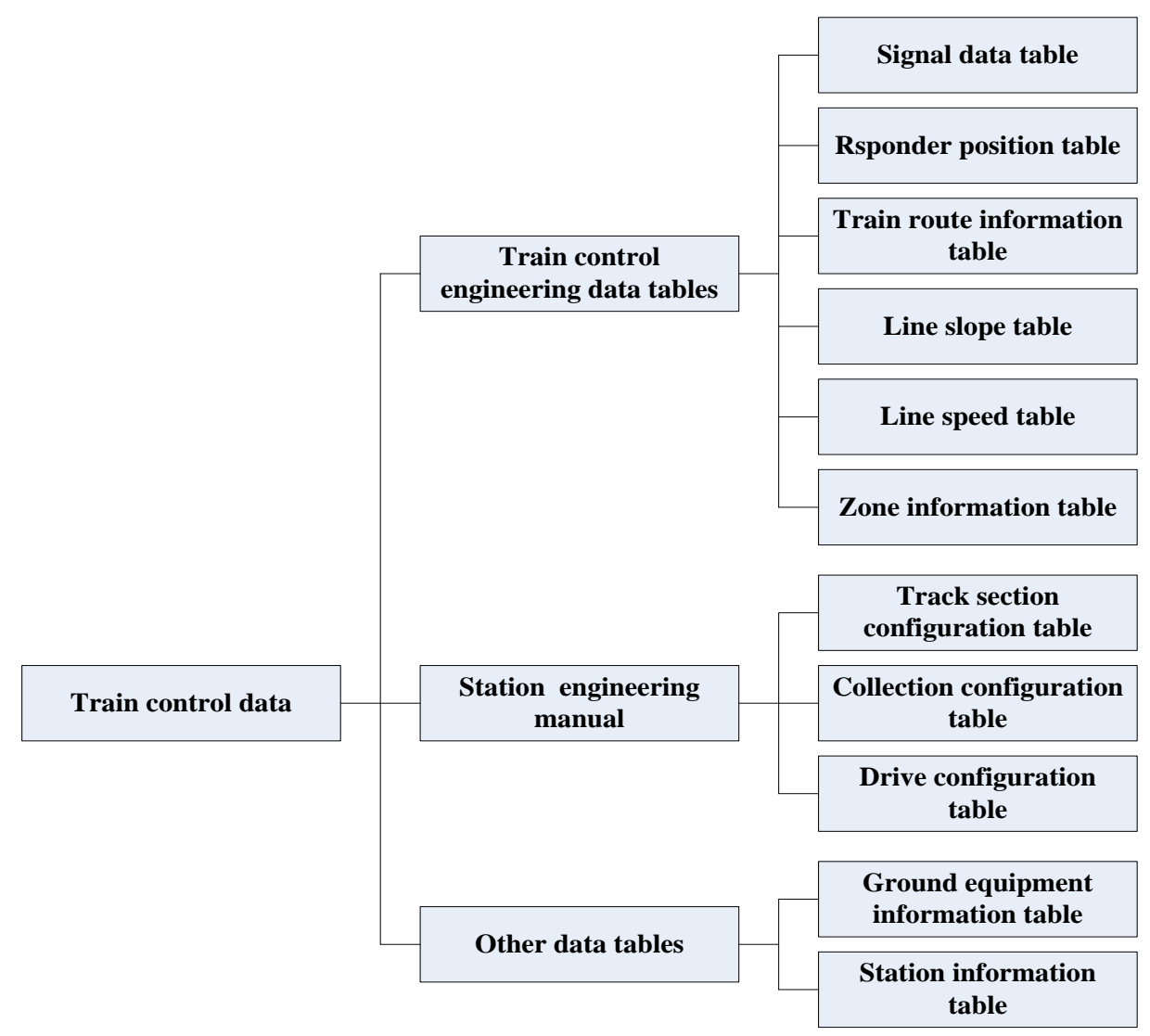

Fig. 2 Main structure of train control system's data

\section{Data Generation Method for Train Control System}

Data Partitioning for Train Control System. Based on the generation method, train control system's data is divided into static data and dynamic data. The static data includes some basic information of the line. Such as line length, position and curvature of the curve, line slope, line speed, the location of the transponder, phase separation, the location of the insulation section, line fault information, foreign matter intrusion information, etc. The dynamic data include the signal display, track circuit code, the interlocking information, etc. All these dynamic data are changing with the location of the train[3].

Static Data of Train Control System. Static data of train control system is provided by the railway administration and research institute which designs railway signal system. They compile the 
static data by comparing the scene of actual situation and the design of line. The static data is stored in a static database[4]. In order to facilitate the radio block center calculating the driving license and on-board ATP device protecting the running train. By testing train running status including train position, train speed, the length of the train and the model of train, ground equipment extract the information of the road in front from the static database. Because the train control system's data once has a mistake, you can not make the train running safety. So in the process of establishing a static database, in particular, we should pay attention to the accuracy of the data.

Dynamic Data of Train Control System. In the process of generating dynamic data, we should consider the relationship between the trains and the way of block division. Take the three-aspect automatic block for example. While there are three free block section in front of the running train, the signal will show a green light. The sequence of the front signal display is green, yellow and red. Take the four-aspect automatic block for example. While there are four free block section in front of the running train, the signal will show a green light. The sequence of the front signal display is green, yellow-green, yellow and red. In this research, we use the latter, because we focus on the CTCS-3 train control system. In the data of track circuit, we should take the relation between the train and the route into consideration. There are four circumstances: the mainline and its connection between arriving route and departing route, the lateral line and its connection between arriving route and departing route, the mainline and its connection between arriving route and departing route on the guide mode and the lateral line and its connection between arriving route and departing route on the guide mode. All these circumstances have the different track circuit codes. According to the specific circumstances, we should select the right coding rules. Interlocking information mostly considers about the space position of the trains. If the mainline is occupied and the train needs to pass through the station by lateral line, the turnout of the approaching interlock should be in the reverse position. And the conflicting route signals should show in red. If the mainline is free, the turnout of the approaching interlock should be in the original position, and the conflicting route signals should show in green.

\section{Summary}

The research mainly bases on the CTCS-3 train control system and analysis the requirements and configuration of train control data. The research also gives the generation method of the train control data. It lays a theoretical foundation for the development of simulation software for the data generation of train control system.

\section{Acknowledgments}

The research has been funded by the National Natural Science Foundation of China (71131001).

The research has been funded by the National Natural Science Youth Foundation of China(71201007).

The research has been funded by the National Natural Science Foundation of China(71571015).

Corresponding Author: Quan-xin Sun, male , doctoral supervisor. Email: qxsun@bjtu.edu.cn.

\section{References}

[1] Wei-zhong Huang, Xue-sheng Ji. Several crucial techniques for the high speed adaptability of CTCS-3 train control on-board equipment[J]. China Railway Science, 2010, 31(3): 87-92.

[2] San-yuan Sheng, Jian-hua WANG. Application of federated Kalman filter in multisensor information fusion[J]. Radar\&ECM, 2002, (1): 27-33.

[3] Chung-Yen Chang. A survey of data protection technologies. Electro Information Technology, 2005 IEEE International Conference on 22-25 May 2005

[4] Information on http://www.aeif.org/ccm/doclist.asp 